\title{
General mapping of multiqudit entanglement conditions to nonseparability indicators for quantum-optical fields
}

\author{
Junghee Ryu $\odot,{ }^{1}$ Bianka Woloncewicz, ${ }^{2}$ Marcin Marciniak $\odot,{ }^{3}$ Marcin Wieśniak, ${ }^{3,2}$ and Marek Żukowski ${ }^{2}$ \\ ${ }^{1}$ Centre for Quantum Technologies, National University of Singapore, 3 Science Drive 2, 117543 Singapore, Singapore \\ ${ }^{2}$ International Centre for Theory of Quantum Technologies (ICTQT), University of Gdansk, 80-308 Gdansk, Poland \\ ${ }^{3}$ Institute of Theoretical Physics and Astrophysics, Faculty of Mathematics, Physics and Informatics, \\ University of Gdańsk, 80-308 Gdańsk, Poland
}

(Received 24 April 2019; published 19 December 2019)

\begin{abstract}
We show that any multiqudit entanglement witness leads to a nonseparability indicator for quantum optical fields, which involves intensity correlations. We get, e.g., necessary and sufficient conditions for intensity or intensity-rate correlations to reveal polarization entanglement. We also derive separability conditions for experiments involving multiport interferometers, now feasible with integrated optics. We show advantages of using intensity rates rather than intensities, e.g., a mapping of the Bell inequalities to ones for optical fields. The results have implication for studies of nonclassicality of "macroscopic" systems of undefined or uncontrollable number of "particles."
\end{abstract}

DOI: 10.1103/PhysRevResearch.1.032041

Nonclassicality due to entanglement initially was studied using quantum optical multiphoton interferometry, see, e.g., Ref. [1]. The experiments were constrained to defined photon number states, e.g., the two-photon polarization singlet [2]. This includes Greenberger-Horne-Zeilinger (GHZ) [3] inspired multiphoton interference, with an interpretation that each detection event signals one photon. Spurious events of higher photon number counts contributed to a lower interferometric contrast. Still, states of undefined photon numbers, e.g., the squeezed vacuum, can be entangled [4-6].

This form of entanglement of quantum optical fields served, e.g., to show that a strongly pumped two-mode (bright) squeezed state allows one to directly refute the ideas of EPR [7], as it approximates their state, and a form of Bell's theorem can be shown for it [4]. The trick was to use displaced parity observables. Recently, it has been shown that this is also possible for four-mode bright squeezed vacuum [8], which can be produced via type II parametric down-conversion, see, e.g., Refs, [5,6]. In this case, the state approximates a tensor product of two EPR states, and interestingly can also be thought of as a polarization "supersinglet" of undefined photon numbers [9]. The approach of Ref. [8] used (effectively) intensity observables, which are less experimentally cumbersome.

With the birth of quantum information science and technology, entanglement became a resource. We have an extended literature on detection of entanglement for systems of finite dimensions, essentially "particles", see e.g., Ref. [10]. It is

Published by the American Physical Society under the terms of the Creative Commons Attribution 4.0 International license. Further distribution of this work must maintain attribution to the author(s) and the published article's title, journal citation, and DOI. well known that not all entangled states violate Bell inequalities. Still there is theory of entanglement indicators, called usually witnesses, which allow to detect entanglement, even if a given state for finite-dimensional systems (essentially, qudits) does not violate any known Bell inequalities. The case of two-mode entanglement for optical fields was studied in trailblazing papers $[11,12]$, which discussed "two-party continuous variable systems," and with a direct quantum optical formalism in Ref. [13]. The entanglement conditions reached in the papers did not involve intensity correlations.

An entanglement condition for four-mode fields, which was borrowing ideas from two spin-1/2 (two-qubit) correlations, involved correlations Stokes operators and was first discussed in Ref. [5]. The resulting indicator was used to measure efficiency of an "entanglement laser." The output of the "laser" was bright four-mode vacuum. We shall present here the most extensive generalization of such an approach, i.e., entanglement indicators for optical fields which are derivatives of multiqudit entanglement witnesses involving intensity correlations. In Ref. [14], we give examples of entanglement conditions based on such an approach. Some of them are more tight versions of the entanglement conditions mentioned above.

As a growing part of the experimental effort is now directed at nonclassical features of bright (intensive "macroscopic") beams of light, e.g., Refs. [15-21] so the time is ripe for a comprehensive study of such entanglement conditions. All that may lead to some new schemes in quantum communication and quantum cryptography, perhaps on the lines of Ref. [9]. The emergence of integrated optics allows now to construct stable multiport interferometers [22-29], and is our motivation of going beyond two times mode case.

We present a theory of mapping multiqudit entanglement witnesses [10] into entanglement indicators for quantum optical fields, which employ intensity correlations or correlations 


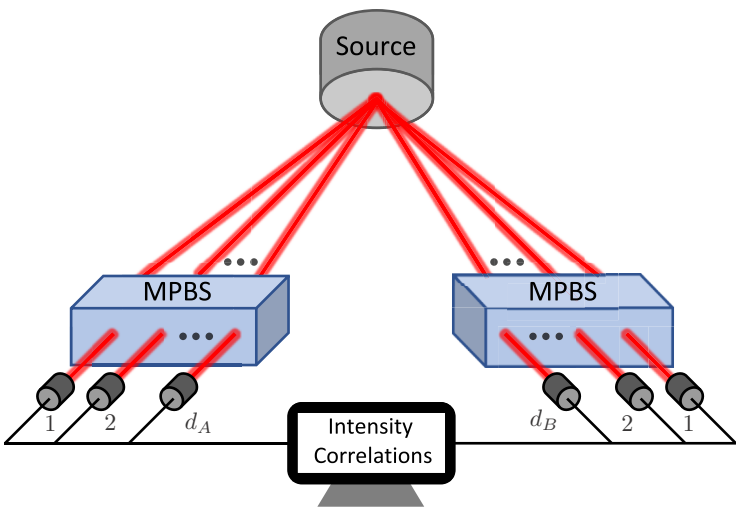

FIG. 1. The experiments (two parties). Two multimode beams propagate to two spatially separated measurement stations. Each station consists of a $d$ input $d$ output tunable multiport beamsplitterinterferometer (MPBS) and detectors at its outputs. For polarization measurements put $d_{A}=d_{B}=2$, and treat the paths as polarization modes.

of intensity rates. By intensity rates we mean the ratio of intensity at a given local detector and the sum of intensities at all local detectors (in some case the second approach leads to better entanglement detection). The method may find applications also in studies of nonclassicality of correlations in "macroscopic" many-body quantum systems of undefined or uncontrollable number of constituents, e.g., Bose-Einstein condensates [30], other specific states of cold atoms [31,32].

The essential ideas are presented for polarization measurements by two observers and the most simple model of intensity observable: photon number in the observed mode. Next, we present further generalization of our approach, and examples employing specific indicators involving intensity correlations for unbiased multiport interferometers. We discuss generalizations to multiparty entanglement indicators. We show that the use of rates leads to a modification of quantum optical Glauber correlation functions, which gives a new tool for studying nonclassicality, and that it also gives a general method of mapping standard Bell inequalities into ones for optical fields.

We discuss spatially separated stations, $X=A, B, \ldots$ with (passive) interferometers of $d_{X}$ input and output ports, Fig. 1. In each output, there is a detector which measures intensity. One can assume either a pulsed source, sources acting synchronously $[33,34]$ or that the measurement is performed within a short time gate. Each time gate, or pulsed emission, is treated as a repetition of the experiment building up averages of observables.

Stokes parameters. For the description of polarization of light, the standard approach uses Stokes parameters. Using the photon numbers they read $\left\langle\hat{\Theta}_{j}\right\rangle=\left\langle\hat{a}_{j}^{\dagger} \hat{a}_{j}-\hat{a}_{j_{\perp}}^{\dagger} \hat{a}_{j_{\perp}}\right\rangle$, where $j, j_{\perp}$ denote a pair of orthogonal polarizations of one of three mutually unbiased polarization bases $j=1,2,3$, e.g., $\{H, V\},\left\{45^{\circ},-45^{\circ}\right\},\{R, L\}$. The zeroth parameter $\left\langle\hat{\Theta}_{0}\right\rangle$ is the total intensity: $\langle\hat{N}\rangle=\left\langle\hat{a}_{j}^{\dagger} \hat{a}_{j}+\hat{a}_{j_{\perp}}^{\dagger} \hat{a}_{j_{\perp}}\right\rangle$. Alternative normalized Stokes observables were studied by some of us [35-37]. They were first introduced in Ref. [38], however a different technical approach was used. Following Ref. [35], one can put $\left\langle\hat{S}_{j}\right\rangle=\left\langle\hat{\Pi} \frac{\left(\hat{a}_{j}^{\dagger} \hat{a}_{j}-\hat{a}_{j_{\perp}}^{\dagger} \hat{a}_{j_{\perp}}\right)}{\hat{N}} \hat{\Pi}\right\rangle$, and $\left\langle\hat{S}_{0}\right\rangle=\langle\hat{\Pi}\rangle$, where $\hat{\Pi}=$ $\mathbb{1}-|\Omega\rangle\langle\Omega|$ and $|\Omega\rangle$ is the vacuum state for the considered modes, $\hat{a}_{j}|\Omega\rangle=\hat{a}_{j_{1}}|\Omega\rangle=0$. Operationally, in the $r$ th run of an experiment, we register photon numbers in the two exits of a polarization analyzer, $n_{j}^{r}$ and $n_{j_{\perp}}^{r}$, and divide their difference by their sum. If $n_{j}^{r}+n_{j_{\perp}}^{r}=0$, the value is put as zero. This does not require any additional measurements, only the data are differently processed than in the standard approach. In Refs. [35-37], examples of the two-party entanglement conditions and Bell inequalities using normalized Stokes operators were given. Here we present a general approach.

Map from two-qubit entanglement witnesses to entanglement indicators for fields involving Stokes parameters. Pauli operators $\vec{\sigma}=\left(\hat{\sigma}_{1}, \hat{\sigma}_{2}, \hat{\sigma}_{3}\right)$ and $\hat{\sigma}_{0}=\mathbb{1}$ form a basis in the real space of one-qubit observables. Thus any two-qubit entanglement witness $\hat{W}$ has the following expansion: $\hat{W}=$ $\sum_{\mu, \nu} w_{\mu \nu} \hat{\sigma}_{\mu}^{A} \otimes \hat{\sigma}_{\nu}^{B}$, where $\mu, v=0,1,2,3$ and $w_{\mu \nu}$ are real coefficients. We have $\langle\hat{W}\rangle_{\text {sep }} \geqslant 0$, where $\langle\cdot\rangle_{\text {sep }}$ denotes an average for a separable state. We will show that with each witness $\hat{W}$ one can associate entanglement indicators for polarization measurements involving correlations of Stokes observables for quantum optical fields. The maps are $\hat{\sigma}_{\mu}^{A} \otimes$ $\hat{\sigma}_{v}^{B} \rightarrow \hat{S}_{\mu}^{A} \hat{S}_{v}^{B}$ and $\hat{\sigma}_{\mu}^{A} \otimes \hat{\sigma}_{v}^{B} \rightarrow \hat{\Theta}_{\mu}^{A} \hat{\Theta}_{v}^{B}$, and they link $\hat{W}$ with its quantum optical analogues $\hat{\mathcal{W}}_{S}=\sum_{\mu, \nu} w_{\mu \nu} \hat{S}_{\mu}^{A} \hat{S}_{v}^{B}$, and $\hat{\mathcal{W}}_{\Theta}=$ $\sum_{\mu, \nu} w_{\mu \nu} \hat{\Theta}_{\mu}^{A} \hat{\Theta}_{\nu}^{B}$, which fulfill $\left\langle\hat{\mathcal{W}}_{S}\right\rangle_{\text {sep }} \geqslant 0$ and $\left\langle\hat{\mathcal{W}}_{\Theta}\right\rangle_{\text {sep }} \geqslant 0$. The proof goes as follows.

Normalized Stokes operators case. It is enough to prove that for any mixed state $\varrho$ one can find a $4 \times 4$ density matrix $\hat{\mathfrak{R}}_{\varrho}^{A B}$ for a pair of qubits, such that

$$
\frac{\left\langle\hat{\mathcal{W}}_{S}\right\rangle_{\varrho}}{\left\langle\hat{\Pi}^{A} \hat{\Pi}^{B}\right\rangle_{\varrho}}=\operatorname{Tr} \hat{W} \hat{\mathfrak{R}}_{\varrho}^{A B} .
$$

First, we show that (1) holds for any pure state $\left|\psi^{A B}\right\rangle$.

Let us denote the polarization basis $H$ and $V$ as $\hat{x}_{H}=$ $\hat{x}_{1}$ and $\hat{x}_{V}=\hat{x}_{2}$. Normalized Stokes operators in arbitrary direction can be put as $\vec{m} \cdot \vec{S}^{X}$, where $\vec{m}$ is an arbitrary unit real vector, or in the matrix form $\sum_{k l} \hat{\Pi}^{X} \frac{\hat{x}_{k}^{\dagger}(\vec{m} \cdot \vec{\sigma})_{k l} \hat{x}_{l}}{\hat{N}^{X}} \hat{\Pi}^{X}$, with $\hat{x}=\hat{a}$ or $\hat{b}$ depending on the beam $X$, whereas $\hat{S}_{0}^{X}$ reads $\sum_{k l} \hat{\Pi}^{X} \frac{\hat{x}_{k}^{\dagger} \delta_{k} \hat{x}_{l}}{\hat{N}^{X}} \hat{\Pi}^{X}$. We introduce a set of states

$$
\left|\Psi_{k m}^{A B}\right\rangle=\hat{a}_{k} \hat{b}_{m} \frac{1}{\sqrt{\hat{N}^{A} \hat{N}^{B}}} \hat{\Pi}^{A} \hat{\Pi}^{B}\left|\psi^{A B}\right\rangle,
$$

where $k, m \in\{1,2\}$. This allows us to put

$$
\begin{aligned}
\left\langle\psi^{A B}\left|\hat{S}_{\mu}^{A} \hat{S}_{v}^{B}\right| \psi^{A B}\right\rangle & =\sum_{k, l=1}^{2} \sum_{m, n=1}^{2} \sigma_{\mu}^{k l} \sigma_{v}^{m n}\left\langle\boldsymbol{\Psi}_{k m}^{A B} \mid \boldsymbol{\Psi}_{l n}^{A B}\right\rangle \\
& =\operatorname{Tr} \hat{\sigma}_{\mu}^{A} \otimes \hat{\sigma}_{v}^{B} \hat{R}_{\psi}^{A B},
\end{aligned}
$$

where the matrix elements of $\hat{R}_{\psi}^{A B}$ are $\left\langle\Psi_{k m}^{A B} \mid \Psi_{l n}^{A B}\right\rangle$. As a Gramian matrix, $\hat{R}_{\psi}^{A B}$ is positive. Except for $\left|\psi^{A B}\right\rangle$ describing vacuum at one or both sides, we have $0<\operatorname{Tr} \hat{R}_{\psi}^{A B}=$ $\left\langle\Pi^{A} \hat{\Pi}^{B}\right\rangle \leqslant 1$. Thus, $\hat{\mathfrak{R}}_{\psi}^{A B}=\hat{R}_{\psi}^{A B} /\left\langle\hat{\Pi}^{A} \hat{\Pi}^{B}\right\rangle$ is an admissible density matrix of two qubits.

For mixed states $\varrho$, i.e., convex combinations of $\left|\psi_{\lambda}^{A B}\right\rangle$ 's with weights $p_{\lambda}$, one gets $\hat{R}_{\varrho}^{A B}=\sum_{\lambda} p_{\lambda} \hat{R}_{\lambda}^{A B}$ which is 
positive definite, and its trace is $\sum_{\lambda} p_{\lambda} \operatorname{Tr} \hat{R}_{\lambda}^{A B} \leqslant 1$. Thus after the re-normalization one gets a proper two-qubit density matrix $\hat{\mathfrak{R}}_{\varrho}^{A B}$. As purity of a field state $\left|\psi_{\lambda}^{A B}\right\rangle$ does not warrant that the corresponding $\hat{R}_{\lambda}^{A B}$ is a projector, $\hat{\mathfrak{R}}_{\varrho}^{A B}$ does not have to have the same convex expansion coefficients in terms of pure two-qubit states, as $\varrho$ in terms of $\left|\psi_{\lambda}^{A B}\right\rangle$ 's.

For any separable pure state of two optical beams $\left|\psi^{A B}\right\rangle_{\text {prod }}$, defined as $F_{A}^{\dagger} F_{B}^{\dagger}|\Omega\rangle$, where $F_{X}^{\dagger}$ is a polynomial function of creation operators for beam (modes) $X$, and $|\Omega\rangle$ is the vacuum state of both beams, the matrix $\hat{R}^{A B}$ factorizes: $\hat{R}^{A B}=\hat{R}^{A} \hat{R}^{B}$. Simply, ${ }^{p r o d}\left\langle\Psi_{k m}^{A B} \mid \Psi_{l n}^{A B}\right\rangle_{\text {prod }}$ factorizes to $\left\langle\Psi_{k}^{A} \mid \Psi_{l}^{A}\right\rangle\left\langle\boldsymbol{\Psi}_{m}^{B} \mid \boldsymbol{\Psi}_{n}^{B}\right\rangle$, where $\left\langle\boldsymbol{\Psi}_{k}^{X} \mid \boldsymbol{\Psi}_{l}^{X}\right\rangle$ are elements of matrix $\hat{R}^{X}$ and $\left|\Psi_{l}^{X}\right\rangle=\hat{x}_{l} \frac{1}{\sqrt{\hat{N}^{X}}} \hat{\Pi}^{X} F_{X}^{\dagger}|\Omega\rangle$. As $\left\langle\Omega\left|F_{X} \hat{\Pi}^{X} F_{X}^{\dagger}\right| \Omega\right\rangle^{-1} \hat{R}^{X}$ can be shown to be a qubit density matrix and $\langle\hat{W}\rangle_{\text {sep }} \geqslant 0$, therefore for pure separable states of the optical beams $\left\langle\hat{\mathcal{W}}_{S}\right\rangle_{\text {prod }} \geqslant 0$. Obviously, $\left\langle\hat{\mathcal{W}}_{S}\right\rangle_{\text {sep }} \geqslant 0$ also for all mixed separable states.

Standard Stokes operators case. Any standard Stokes operator can be put as $\vec{m} \cdot \vec{\Theta}^{X}=\sum_{k l} \hat{x}_{k}^{\dagger}(\vec{m} \cdot \vec{\sigma})_{k l} \hat{x}_{l}$. We introduce state vectors $\left|\boldsymbol{\Phi}_{j k}^{A B}\right\rangle=\hat{a}_{j} \hat{b}_{k}\left|\psi^{A B}\right\rangle$. One has

$$
\left\langle\psi^{A B}\left|\hat{\Theta}_{\mu}^{A} \hat{\Theta}_{v}^{B}\right| \psi^{A B}\right\rangle=\operatorname{Tr} \hat{\sigma}_{\mu}^{A} \hat{\sigma}_{\nu}^{B} \hat{P}^{A B},
$$

where the matrix $\hat{P}^{A B}$ has entries $\left\langle\boldsymbol{\Phi}_{k m}^{A B} \mid \boldsymbol{\Phi}_{l n}^{A B}\right\rangle$, it is positive definite, and its trace is $\left\langle\hat{N}_{A} \hat{N}_{B}\right\rangle$. Thus $\hat{\mathfrak{P}}^{A B}=\hat{P}^{A B} /\left\langle\hat{N}^{A} \hat{N}^{B}\right\rangle$ is an admissible two-qubit density matrix, and one has $\left\langle\hat{\mathcal{W}}_{\Theta}\right\rangle_{\varrho} /\left\langle\hat{N}^{A} \hat{N}^{B}\right\rangle_{\varrho}=\operatorname{Tr} \hat{W} \hat{\mathfrak{P}}_{\varrho}^{A B}$. All that leads to $\left\langle\hat{\mathcal{W}}_{\Theta}\right\rangle_{\text {sep }} \geqslant 0$. Note that, for a general state $\hat{\mathfrak{R}}_{\varrho}^{A B}$ does not have to be equal to $\hat{\mathfrak{P}}_{\varrho}^{A B}$. Still $\hat{\mathfrak{R}}^{A B}=\hat{\mathfrak{P}}^{A B}$ for states of defined photon numbers in both beams.

Reverse map. Any linear separability condition expressible in terms of correlation functions of normalized Stokes parameters reads $\sum_{\mu \nu} \omega_{\mu \nu}\left\langle\hat{S}_{\mu}^{A} \hat{S}_{\nu}^{B}\right\rangle_{\text {sep }} \geqslant 0$. As two-photon states, with one at $\mathrm{A}$ and the other at $\mathrm{B}$, are possible field states, thus for any separable such state we must have $\sum_{\mu \nu} \omega_{\mu \nu}\left\langle\hat{S}_{\mu}^{A} \hat{S}_{v}^{B}\right\rangle_{s e p-2-p h} \geqslant 0$. This is algebraically equivalent to $\sum_{\mu \nu} \omega_{\mu \nu}\left\langle\hat{\sigma}_{\mu} \otimes \hat{\sigma}_{\nu}\right\rangle_{\text {sep }} \geqslant 0$, for any two-qubit state. We get an entanglement witness. Therefore, we have an isomorphism. Similar proof applies to standard Stokes observables.

Examples. In Ref. [14], we show some examples of entanglement indicators which can be derived with the above method. This includes a necessary and sufficient conditions for detection of entanglement of two optical beams with correlations of Stokes parameters of the two considered kinds.

Detection losses. Consider the usual model of losses: a perfect detector in front of which is a beamsplitter of transmission amplitude $\eta$, with the reflection channel describing the losses. Then, $\left\langle\hat{\Theta}_{\mu}^{A} \hat{\Theta}_{v}^{B}\right\rangle$ scales down as $\eta^{A} \eta^{B}$ (see Sec. II in Ref. [14]), where $\eta^{X}$ for $X=A, B$ is the local detection efficiency. We have a full resistance of entanglement detection, using any $\hat{\mathcal{W}}_{\Theta}$, with respect to such losses. A different character of losses may lead to threshold efficiencies.

For the normalized Stokes parameters, it is enough to consider only pure states, because mixed ones, as convex combinations of such, cannot introduce anything new in entanglement conditions linear with respect to the density matrix. Any pure state is a superposition of Fock states $|F\rangle=$ $\left|n_{i}^{A}, n_{i_{\perp}}^{A}, n_{j}^{B}, n_{j_{\perp}}^{B}\right\rangle$, where $n_{i}^{X}$ denotes the number of $i$ polarized photons in beam $X$, and $\hat{S}_{\mu}^{A} \hat{S}_{v}^{B}$ are diagonal with respect to the Fock basis related with them. Thus, the dependence on efficiencies of the value of an entanglement indicator, in the case of a pure state, depends on the behavior of its Fock components. One can show, see Sec. II in Ref. [14], that $\left\langle F_{\eta}\left|\hat{S}_{\mu}^{A} \hat{S}_{v}^{B}\right| F_{\eta}\right\rangle=H_{F}\left\langle F\left|\hat{S}_{\mu}^{A} \hat{S}_{v}^{B}\right| F\right\rangle$, where $\left|F_{\eta}\right\rangle$ is the state $|F\rangle$ after the above described losses in both channels, and $H_{F}=$ $\left\langle F_{\eta}\left|\hat{S}_{0}^{A} \hat{S}_{0}^{B}\right| F_{\eta}\right\rangle$, which reads $\prod_{X=A, B}\left[1-\left(1-\eta^{X}\right)^{m^{X}}\right]$, where $m^{X}$ is the total number of photons in channel $X$, before the losses. Expanding $|F\rangle$ in terms of Fock states with respect to different polarizations than $i, i_{\perp}$ and $j, j_{\perp}$, does not change the values of $m^{X}$, and thus the formula stays put for any indices. Again we have a strong resistance of the entanglement indicators with respect to losses. Especially for states with high photon numbers, the entanglement conditions based on normalized Stokes parameters, may be more resistant to losses, because $0<\eta<1$, one has $\eta<1-(1-\eta)^{n}$.

Multiparty case. Consider three parties, and the case of indicators of genuine three-beam entanglement. Any genuine three-qubit entanglement witness $\hat{W}^{(3)}$ has the property that it is positive for pure product three-qubit states $|\xi\rangle_{A B, C}=$ $|\psi\rangle_{A B}|\phi\rangle_{C}$, for similar ones with qubits permuted, and for all convex combinations of such states. With any pure partial product state of the optical beams, e.g., $|\Xi\rangle_{A B, C}=F_{A B}^{\dagger} F_{C}^{\dagger}|\Omega\rangle$, where $F_{A B}^{\dagger}$ is an operator built of creation operators for beams $A$ and $B$, etc., one can associate, in a similar way as above, a partially factorizable three-qubit density matrix $\hat{\mathfrak{R}}_{\psi}^{A B} \hat{\mathfrak{R}}_{\phi}^{C}$. Thus the homomorphism works. Generalizations are obvious.

General theory. Consider a beam of $d_{A}$ quantum optical modes propagating toward a measuring station $A$, and a beam of $d_{B}$ modes toward station $B$. We associate with the situation a $d_{A} \times d_{B}$-dimensional Hilbert Space, $\mathbb{C}^{d_{A}} \otimes \mathbb{C}^{d_{B}}$, which contains pure states of a pair of qudits of dimensions $d_{A}$ and $d_{B}$. For $X=A, B$, let $\hat{V}_{i}^{X}$, with $i=1, \ldots, d_{X}^{2}$, be an orthonormal, i.e., $\operatorname{Tr} \hat{V}_{i}^{X} \hat{V}_{j}^{X}=\delta_{i j}$, Hermitian basis of the space of Hermitian operators acting on $\mathbb{C}^{d_{X}}$. Therefore products $\hat{V}_{i}^{A} \otimes \hat{V}_{j}^{B}$ form an orthonormal basis of the space of Hermitian operators acting on $\mathbb{C}^{d_{A}} \otimes \mathbb{C}^{d_{B}}$. Thus any entanglement witness for the pair of qudits, $\hat{W}$, can be expanded into

$$
\hat{W}=\sum_{j=1}^{d_{A}^{2}} \sum_{k=1}^{d_{B}^{2}} w_{j k} \hat{V}_{j}^{A} \otimes \hat{V}_{k}^{B},
$$

with real $w_{j k}$. The optimal expansion (with the minimal number of terms) is to use a Schmidt basis for $\hat{W}$.

Each $\hat{V}_{j}^{X}$ can be decomposed to a linear combination of its spectral projections linked with their respective eigenbases, $\left|x_{l}^{(j)}\right\rangle$, where $x=a$ or $b$ consistently with $X$ and $l=$ $1, \ldots, d_{X}$. If one fixes a certain pair of bases in $\mathbb{C}^{d_{A}}$ and $\mathbb{C}^{d_{B}}$ as "computational ones," i.e., starting ones, denoted as $\left|l_{x}\right\rangle$, one can always find local unitary matrices $U^{X}(j)$ such that $U^{X}(j)\left|l_{x}\right\rangle=\left|x_{l}^{(j)}\right\rangle$. The construction of Reck et al. [39] fixes (phases in) a local multiport interferometer, which performs such a transformation. We shall call such interferometers $U^{X}(j)$ ones. In the case of field modes a passive interferometer performs the following mode transformation: $\sum_{k} U^{X}(j)_{l k} \hat{x}_{k}^{\dagger}=\hat{x}_{l}^{\dagger}(j)$, where $\hat{x}_{l}^{\dagger}(j)$ is the photon creation operator in the $l$ th exit mode of interferometer $U^{X}(j)$. 
A two-party entanglement witness $\hat{\mathcal{W}}_{R}$ for optical fields, which uses correlations of intensity rates behind pairs of $U^{X}(j)$ interferometers can be constructed as follows. For the output $l_{x}$ of an interferometer, one defines rate observables as $\hat{r}_{l_{x}}=\hat{\Pi}^{X} \frac{\hat{l}_{l_{x}}}{\hat{N}^{X}} \hat{\Pi}^{X}$, where $\hat{N}^{X}=\sum_{l_{x}=1}^{d_{X}} \hat{n}_{l_{x}}$. The witness $\hat{W}$ expanded in terms of the computational basis:

$$
\hat{W}=\sum_{k, m}^{d_{A}} \sum_{l, n}^{d_{B}} w_{k l m n}\left|k_{a}, l_{b}\right\rangle\left\langle m_{a}, n_{b}\right|,
$$

allows us to form an entanglement witness for fields:

$$
\hat{\mathcal{W}}_{R}=\sum_{k, m} \sum_{l, n} w_{k l m n} \hat{\Pi}^{A} \hat{\Pi}^{B} \frac{\hat{a}_{k}^{\dagger} \hat{b}_{l}^{\dagger} \hat{a}_{m} \hat{b}_{n}}{\hat{N}^{A} \hat{N}^{B}} \hat{\Pi}^{A} \hat{\Pi}^{B} .
$$

For any pure state of the quantum beams $|\Psi\rangle$

$$
\frac{\left\langle\Psi\left|\hat{\mathcal{W}}_{R}\right| \Psi\right\rangle}{\left\langle\Psi\left|\hat{\Pi}^{A} \hat{\Pi}^{B}\right| \Psi\right\rangle}=\operatorname{Tr} \hat{W} \hat{\mathcal{R}},
$$

where the matrix $\hat{\mathcal{R}}$ has elements $r_{k l m n}$

$$
r_{k l m n}=\frac{1}{\left\langle\Psi\left|\hat{\Pi}^{A} \hat{\Pi}^{B}\right| \Psi\right\rangle}\left\langle\Psi\left|\hat{\Pi}^{A} \hat{\Pi}^{B} \frac{\hat{a}_{k}^{\dagger} \hat{b}_{l}^{\dagger} \hat{a}_{m} \hat{b}_{n}}{\hat{N}^{A} \hat{N}^{B}} \hat{\Pi}^{A} \hat{\Pi}^{B}\right| \Psi\right\rangle .
$$

Using a generalization of the earlier derivations, one can show that $\hat{\mathcal{R}}$ is a two-qudit density matrix, and so on.

The actual measurements, to be correlations of local ones, should be performed using the sequence of pairs of $U^{X}(j)$ interferometers, which enter the expansion of the two-qudit entanglement witness (5). In the entanglement indicator the rates at output $x_{l}(j)$ of the given local interferometer $U^{X}(j)$ are multiplied by the respective eigenvalue of $\hat{V}_{j}^{X}$ related with the eigenstate $\left|x_{l}^{(j)}\right\rangle$.

To get an entanglement witness for intensities $\hat{\mathcal{W}}_{I}$ we take $\hat{W}$ and replace the computational basis kets and bras by suitable creation and annihilation operators:

$$
\hat{\mathcal{W}}_{I}=\sum_{k, m}^{d_{A}} \sum_{l, n}^{d_{B}} w_{k l m n} \hat{a}_{k}^{\dagger} \hat{b}_{l}^{\dagger} \hat{a}_{m} \hat{b}_{n}
$$

For any pure state of the quantum beams $|\Psi\rangle$, one has $\frac{\left\langle\Psi\left|\hat{\mathcal{W}}_{I}\right| \Psi\right\rangle}{\left\langle\Psi\left|\hat{N}_{A} \hat{N}_{B}\right| \Psi\right\rangle}=\operatorname{Tr} \hat{W} \hat{\mathcal{P}}$, where the matrix $\hat{\mathcal{P}}$ has elements $\frac{1}{\left\langle\Psi\left|\hat{N}_{A} \hat{N}_{B}\right| \Psi\right\rangle}\left\langle\Psi\left|\hat{a}_{k}^{\dagger} \hat{b}_{l}^{\dagger} \hat{a}_{m} \hat{b}_{n}\right| \Psi\right\rangle$, and has all properties of a twoqudit density matrix.

Example showing further extension to unitary operator bases. Let $d$ be a power of a prime number. Consider $d_{A}=$ $d_{B}=d$ beams experiment (see Fig. 1), with families of $U^{X}(m)$ interferometers which link the computational basis of a qudit with an unbiased basis $m$, belonging to the full set of $d+1$ mutually unbiased ones [40,41]. We introduce a set of unitary observables for a qudit: $\hat{q}_{k}(m)=\sum_{j=1}^{d} \omega^{j k}|j(m)\rangle\langle j(m)|$, with $|j(m)\rangle=U(m)|j\rangle$ and it is the $j$ th member of $m$ th mutually unbiased basis, and $\omega=\exp (2 \pi i / d)$. Operators $\hat{q}_{k}(m) / \sqrt{d}$ with $k=1, \ldots, d-1$ and $m=0, \ldots, d$ and $\hat{q}_{0}(0) / \sqrt{d}$ form an orthonormal basis in the Hilbert-Schmidt space of all $d \times d$ matrices (see Sec. III in Ref. [14]). Thus we can expand any qudit density matrix as

$$
\varrho=\frac{1}{\sqrt{d}}\left[c_{0,0} \hat{q}_{0}(0)+\sum_{m=0}^{d} \sum_{k=1}^{d-1} c_{m, k} \hat{q}_{k}(m)\right],
$$

where $c_{m, k}=\operatorname{Tr} \hat{q}_{k}^{\dagger}(m) \varrho / \sqrt{d}$, and $c_{0,0}=1 / \sqrt{d}$. As the basis observables are unitary the expansion coefficients of an entanglement witness operator in terms of such tensor products of such bases are in general complex. This is no problem for theory, but renders useless a direct application in experiments, as one cannot expect the experimental averages to be real, and thus one has to introduce modifications. Below we present one.

The condition $\operatorname{Tr} \varrho^{2} \leqslant 1$ can be put as

$$
\frac{1}{d}+\frac{1}{d} \sum_{m=0}^{d} \sum_{k=1}^{d-1}\left|\operatorname{Tr} \varrho \hat{q}_{k}(m)\right|^{2} \leqslant 1 .
$$

Thus, applying Cauchy-Schwartz estimate, we get immediately a separability condition for two qudits:

$$
\sum_{m=0}^{d} \sum_{k=1}^{d-1}\left|\operatorname{Tr} \varrho_{\text {sep }}^{A B} \hat{q}_{k}^{A}(m) \hat{q}_{k}^{B^{\dagger}}(m)\right| \leqslant(d-1) .
$$

Our general method defines a Cauchy-Schwartz-like separability condition homomorphic with (13) as

$$
\sum_{m=0}^{d} \sum_{k=1}^{d-1}\left|\left\langle\hat{Q}_{k}^{A}(m) \hat{Q}_{k}^{B \dagger}(m)\right\rangle_{\mathrm{sep}}\right| \leqslant(d-1)\left\langle\hat{\Pi}^{A} \hat{\Pi}^{B}\right\rangle_{\mathrm{sep}},
$$

where

$$
\hat{Q}_{k}^{X}(m)=\sum_{j=1}^{d} \hat{\Pi}^{X} \frac{\omega^{j k} \hat{n}_{j}^{X}(m)}{\hat{N}^{X}} \hat{\Pi}^{X}
$$

Here, $\hat{n}_{j}^{X}(m)=\hat{x}_{j}^{\dagger}(m) \hat{x}_{j}(m)$ is a photon number operator for output mode $j$ of a multiport $m$, at station $X$. For generalized observables based on intensity, one can introduce $\hat{\chi}_{k}(m)=$ $\sum_{j=1}^{d} \omega^{j k} \hat{n}_{j}(m)$ to get the following separability condition:

$$
\sum_{m=0}^{d} \sum_{k=1}^{d-1}\left|\left\langle\hat{\chi}_{k}^{A}(m) \hat{\chi}_{k}^{B^{\dagger}}(m)\right\rangle_{\text {sep }}\right| \leqslant(d-1)\left\langle\hat{N}^{A} \hat{N}^{B}\right\rangle_{\text {sep }} .
$$

Reference [14] presents other examples.

Implications for optical coherence theory. The approach can be generalized further. Let us take as an example Glauber's correlation functions for optical fields, say $G^{(4)}$ in the form of $\left\langle\hat{I}_{A}(\vec{x}, t) \hat{I}_{B}\left(\vec{x}^{\prime}, t^{\prime}\right)\right\rangle$, where the intensity operator has the usual form of $I_{X}(\vec{x}, t)=\hat{F}_{X}^{\dagger}(\vec{x}, t) \hat{F}_{X}(\vec{x}, t)$, with normal ordering requiring that operator $\hat{F}_{X}(\vec{x}, t)$ is built out of local annihilation operators. The idea of normalized Stokes operators suggests the following alternative correlation function $\Gamma^{4}\left(\vec{x}, t ; \vec{x}^{\prime}, t^{\prime}\right)$ given by

$$
\left\langle\Pi^{A} \Pi^{B} \frac{\hat{I}_{A}(\vec{x}, t) \hat{I}_{B}\left(\vec{x}^{\prime}, t^{\prime}\right)}{\int_{a(A)} d \sigma(\vec{x}) \hat{I}_{A}(\vec{x}, t) \int_{a(B)} d \sigma\left(\vec{x}^{\prime}\right) \hat{I}_{B}\left(\vec{x}^{\prime}, t^{\prime}\right)} \Pi^{A} \Pi^{B}\right\rangle,
$$


where $a(X)$ denotes the overall aperture of the detectors in location $X$. Obviously one has $\int_{a(A)} d \sigma(\vec{x}) \int_{a(B)} d \sigma\left(\vec{x}^{\prime}\right) \Gamma^{4}\left(\vec{x}, t ; \vec{x}^{\prime}, t^{\prime}\right)=$ $\left\langle\Pi^{A} \Pi^{B}\right\rangle$, and for fixed $t$ and $t^{\prime}$, one can define

$$
\varrho\left(\vec{x}, \vec{y}, \vec{x}^{\prime} \vec{y}^{\prime}\right)_{t, t^{\prime}}=\left\langle\Pi^{A} \Pi^{B}\right\rangle^{-1}\left\langle\Pi^{A} \Pi^{B} \frac{\hat{F}_{A}^{\dagger}(\vec{y}, t) \hat{F}_{A}(\vec{x}, t) \hat{F}_{B}^{\dagger}\left(\vec{y}^{\prime}, t^{\prime}\right) \hat{F}_{B}\left(\vec{x}^{\prime}, t^{\prime}\right)}{\int_{a(A)} d \sigma(\vec{x}) \hat{I}_{A}(\vec{x}, t) \int_{a(B)} d \sigma\left(\vec{x}^{\prime}\right) \hat{I}_{B}\left(\vec{x}^{\prime}, t^{\prime}\right)} \Pi^{A} \Pi^{B}\right\rangle,
$$

which behaves like a proper two-particle density matrix, provided one constrains the range of $\vec{x}, \vec{y}, \vec{x}^{\prime}, \vec{y}^{\prime}$ to appropriate sets of apertures. As our earlier considerations use simplified forms of (17), it is evident that such correlation functions may help us to unveil nonclassicality in situations in which the standard ones fail, see, e.g., Ref. [8].

Bell inequalities. The above ideas allow one to introduce a general mapping of qudit Bell inequalities to the ones for optical fields. A two-qudit Bell inequality for a final number of local measurement settings $\alpha$ and $\beta$ has the following form:

$$
\begin{aligned}
& \sum_{\alpha \beta} \sum_{i=1}^{d_{A}} \sum_{j=1}^{d_{B}} K_{\alpha \beta}^{i j} P_{i j}(\alpha, \beta) \\
& \quad+\sum_{i=1}^{d_{A}} \sum_{\alpha} N_{\alpha}^{i} P_{i}(\alpha)+\sum_{j=1}^{d_{B}} \sum_{\beta} M_{\beta}^{j} P_{j}(\beta) \leqslant L_{R},
\end{aligned}
$$

where $P_{i j}(\alpha, \beta)$ denotes the probability of the qudits ending up respectively at detectors $i$ and $j$, when the local setting are as indicated, and $\sum_{j} P_{i j}(\alpha, \beta)=P_{i}(\alpha)$ and $P_{j}(\beta)=$ $\sum_{i} P_{i j}(\alpha, \beta)$. The coefficient matrices $K, N, M$ are real, and $L_{R}$ is the maximum value allowed by local realism. The bound is calculated by putting $P_{i j}(\alpha, \beta)=D^{i}(\alpha) D^{j}(\beta)$ and $P_{i}(\alpha)=$ $D^{i}(\alpha), P_{j}(\beta)=D^{j}(\beta)$, with constraints $0 \leqslant D^{i}(\alpha / \beta) \leqslant 1$, and $\sum_{i=1}^{d_{A / B}} D^{i}(\alpha / \beta)=1$. As for a given run of a quantum optical experiment local measured photon intensity rates $r_{i}(\alpha)$ and $r_{j}(\beta)$ satisfy exactly the same constraints. We can replace $P_{i j}(\alpha, \beta) \rightarrow\left\langle r_{i}(\alpha) r_{j}(\beta)\right\rangle_{L R}$, and $P_{i}(\alpha) \rightarrow\left\langle r_{i}(\alpha)\right\rangle_{L R}$, etc., where $\langle.\rangle_{L R}$ is an average in the case of local realism. The bound $L_{R}$ stays put. To get a Bell operator we further replace the above by rate observables $\hat{r}_{i}(\alpha) \hat{r}_{j}(\beta)$, etc. Thus any (multiparty) Bell inequality, see, e.g., Ref. [42], can be useful in quantum optical intensity (rates) correlation experiments. The presented methods for entanglement indicators and the Bell inequalities allow also to get steering inequalities for quantum optics.

Conclusions. We present tools for a construction of entanglement indicators for optical fields, inspired by the vast literature [10] on entanglement witnesses for finite dimensional quantum systems. The indicators would be handy for more intense light beams in states of undefined photon numbers, especially in the emerging field of integrated optics multispatial mode interferometry (see Ref. [14] for examples). One may expect applications in the case of many-body systems, e.g., for an analysis of nonclassicality of correlations in BoseEinstein condensates, like in the ones reported in Ref. [43].

Acknowledgments. The work is part of the ICTQT IRAP project of FNP, financed by structural funds of EU. MZ acknowledges COPERNICUS grant-award and discussions with Maria Chekhova and Harald Weinfurter. JR acknowledges the National Research Foundation, Prime Minister's Office, Singapore, and the Ministry of Education, Singapore, under the Research Centres of Excellence programme, and discussions with Dagomir Kaszlikowski. MW acknowledges NCN Grants No. 2015/19/B/ST2/01999 and No. 2017/26/E/ST2/01008.
[1] J.-W. Pan, Z. B. Chen, C. Y. Lu, H. Weinfurter, A. Zeilinger, and M. Żukowski, Rev. Mod. Phys. 84, 777 (2012).

[2] A. Aspect, P. Grangier, and G. Roger, Phys. Rev. Lett. 49, 91 (1982).

[3] D. M. Greenberger, M. A. Horne, and A. Zeilinger, Bell's Theorem, Quantum Theory, and Conceptions of the Universe, edited by M. Kafatos (Kluwer, Dordrecht, 1989), pp. 69-72.

[4] K. Banaszek and K. Wódkiewicz, Phys. Rev. A 58, 4345 (1998); Acta. Phys. Slov. 49, 491 (1999).

[5] C. Simon and D. Bouwmeester, Phys. Rev. Lett. 91, 053601 (2003).

[6] M. V. Chekhova, G. Leuchs, and M. Żukowski, Opt. Commun. 337, 27 (2015).

[7] A. Einstein, B. Podolsky, and N. Rosen, Phys. Rev. 47, 777 (1935).

[8] K. Rosołek, K. Kostrzewa, A. Dutta, W. Laskowski, M. Wieśniak, and M. Żukowski, Phys. Rev. A 95, 042119 (2017).

[9] G. A. Durkin, C. Simon, and D. Bouwmeester, Phys. Rev. Lett. 88, 187902 (2002).
[10] R. Horodecki, P. Horodecki, M. Horodecki, and K. Horodecki, Rev. Mod. Phys. 81, 865 (2009).

[11] R. Simon, Phys. Rev. Lett. 84, 2726 (2000)

[12] L. M. Duan, G. Giedke, J. I. Cirac, and P. Zoller, Phys. Rev. Lett. 84, 2722 (2000).

[13] M. Hillery and M. S. Zubairy, Phys. Rev. Lett. 96, 050503 (2006)

[14] See Supplemental Material at http://link.aps.org/supplemental/ 10.1103/PhysRevResearch.1.032041 for more details.

[15] T. Sh. Iskhakov, I. N. Agafonov, M. V. Chekhova, and G. Leuchs, Phys. Rev. Lett. 109, 150502 (2012).

[16] B. Kanseri, T. Iskhakov, G. Rytikov, M. Chekhova, and G. Leuchs, Phys. Rev. A 87, 032110 (2013).

[17] H. S. Eisenberg, G. Khoury, G. A. Durkin, C. Simon, and D. Bouwmeester, Phys. Rev. Lett. 93, 193901 (2004).

[18] T. Sh. Iskhakov, K. Y. Spasibko, M. V. Chekhova, and G. Leuchs, New J. Phys. 15, 093036 (2013).

[19] A. Lamas-Linares, J. C. Howell, and D. Bouwmeester, Nature (London) 412, 887 (2001). 
[20] A. Eckstein, A. Christ, P. J. Mosley, and C. Silberhorn, Phys. Rev. Lett. 106, 013603 (2011).

[21] K. Yu. Spasibko, D. A. Kopylov, V. L. Krutyanskiy, T. V. Murzina, G. Leuchs, and M. V. Chekhova, Phys. Rev. Lett. 119, 223603 (2017).

[22] K. Mattle, M. Michler, H. Weinfurter, A. Zeilinger, and M. Żukowski, Appl. Phys. B 60, S111 (1995).

[23] G. Weihs, M. Reck, H. Weinfurter, and A. Zeilinger, Phys. Rev. A 54, 893 (1996).

[24] A. Peruzzo, A. Laing, A. Politi, T. Rudolph and J. L. O'Brien, Nat. Commun. 2, 224 (2011).

[25] T. Meany, M. Delanty, S. Gross, G. D. Marshall, M. J. Steel, and M. J. Withford, Opt. Express 20, 26895 (2012).

[26] B. J. Metcalf et al., Nat. Commun. 4, 1356 (2013).

[27] N. Spagnolo, C. Vitelli, L. Aparo, P. Mataloni, F. Sciarrino, A. Crespi, R. Ramponi, and R. Osellame, Nat. Commun. 4, 1606 (2013).

[28] J. Carolan et al., Science 349, 711 (2015).

[29] C. Schaeff, R. Polster, M. Huber, S. Ramelow, and A. Zeilinger, Optica 2, 523 (2015).

[30] A. Sørensen, L.-M. Duan, J. I. Cirac, and P. Zoller, Nature (London) 409, 63 (2001).

[31] J. Hald, J. L. Sørensen, C. Schori, and E. S. Polzik, Phys. Rev. Lett. 83, 1319 (1999).
[32] A. S. Sørensen and K. Mølmer, Phys. Rev. Lett. 86, 4431 (2001).

[33] M. Żukowski, A. Zeilinger and H. Weinfurter, Ann. NY Acad. Sci. 755, 91 (1995).

[34] R. Kaltenbaek, B. Blauensteiner, M. Żukowski, M. Aspelmeyer, and A. Zeilinger, Phys. Rev. Lett. 96, 240502 (2006).

[35] M. Żukowski, W. Laskowski, and M. Wieśniak, Phys. Rev. A 95, 042113 (2017).

[36] M. Żukowski, W. Laskowski, and M. Wieśniak, Phys. Scr. 91, 084001 (2016).

[37] M. Żukowski, M. Wieśniak, and W. Laskowski, Phys. Rev. A 94, 020102(R) (2016).

[38] Q. Y. He, M. D. Reid, T. G. Vaughan, C. Gross, M. Oberthaler, and P. D. Drummond, Phys. Rev. Lett. 106, 120405 (2011); Q. Y. He, T. G. Vaughan, P. D. Drummond, and M. D. Reid, New J. Phys. 14, 093012 (2012).

[39] M. Reck, A. Zeilinger, H. J. Bernstein, and P. Bertani, Phys. Rev. Lett. 73, 58 (1994).

[40] W. K. Wootters and B. D. Fields, Ann. Phys. 191, 363 (1989).

[41] I. D. Ivanovic, J. Phys. A: Math. Gen. 14, 3241 (1981).

[42] N. Brunner, D. Cavalcanti, S. Pironio, V. Scarani, and S. Wehner, Rev. Mod. Phys. 86, 419 (2014).

[43] R. Schmied, J.-D. Bancal, B. Allard, M. Fadel, V. Scarani, P. Treutlein, and N. Sangouard, Science 352, 441 (2016). 